\title{
Long Noncoding RNA LINC00963 Promotes CDC5L-Mediated Malignant Progression in Gastric Cancer
}

This article was published in the following Dove Press journal: OncoTargets and Therapy

\author{
Hong Zhu \\ Jin-Hai Tang \\ Shi-Meng Zhang \\ Jia-Ping Qian \\ Xin Ling \\ Xiao-Ying Wu \\ Ling-Xia Yang
}

Department of Gastroenterology, Suzhou Ninth People's Hospital, Suzhou, Jiangsu Province, People's Republic of China
Correspondence: Ling-Xia Yang

Email ylxnjq@163.com
Background: Gastric cancer (GC) is a common cancer with high incidence and mortality worldwide. In recent years, accumulating evidence has shown that long noncoding RNAs (lncRNAs) exert critical roles in the development and progression of cancer by acting as a tumor initiator or suppressor. LINC00963 is a newly reported lncRNA related to cancer, and its role in $\mathrm{GC}$ remains unclear.

Materials and Methods: The expression levels of LINC00963, miR-612, and cell division cycle 5-like protein (CDC5L) were measured using quantitative real-time PCR or Western blot. The biological functions of LINC00963, miR-612, and CDC5L in GC cells were analyzed by transwell and proliferation experiments. The expression of CDC5L in patients with GC was evaluated using the Oncomine database. Bone marrow-derived dendritic cells (DCs) were derived from C57BL/6 mice.

Results: LINC00963 expression was higher in GC tissues than in adjacent normal tissues. Similar results were found in GC cell lines and normal human gastric epithelial cells. Upregulation of LINC00963 was related to the poor prognosis of patients with GC. Knockdown of LINC00963 inhibited the proliferation, invasion, and metastasis but promoted the apoptosis of GC cells. Furthermore, silencing of LINC00963 in GC cells significantly suppressed the tumor growth of GC. Bioinformatics analysis indicated that LINC00963 could target miR-612 by functioning as a competing endogenous RNA. The expression of miR-612 decreased in GC tissues and cell lines. Meanwhile, LINC00963 expression was negatively associated with miR-612. CDC5L was a direct target of miR-612. miR-612 suppressed the expression of CDC5L in GC tissues and cells. Moreover, LINC00963 inhibited the differentiation and maturation of DCs by regulating miR-612 expression in DCs.

Conclusion: LINC00963 promoted the progression of GC by competitively binding to miR612 to regulate the expression of $\mathrm{CDC} 5 \mathrm{~L}$ and mediated DC-related anti-tumor immune response. Thus, targeting LINC00963 may be a promising therapeutic strategy for GC.

Keywords: lncRNA LINC00963, gastric cancer, miR-612, CDC5L, dendritic cells

\section{Introduction}

$\mathrm{GC}$ is a common digestive system cancer and the second leading cause of cancerassociated deaths worldwide. ${ }^{1}$ Although the prevalence of GC has been decreasing steadily in recent years, ${ }^{2,3}$ this cancer remains malignant and deadly, particularly in East Asia. ${ }^{4}$ The World Health Organization classified GC into four types: tubular cancer, papillary cancer, mucinous cancer, and poorly cohesive carcinomas. ${ }^{5}$ In the past few years, treatments for GC have achieved great advances, including surgical 
removal, chemotherapy, radiotherapy, and gene-targeted therapy. However, the poor prognosis of patients with GC remains a serious concern. ${ }^{6}$ In addition, patients are diagnosed at the advanced stage of the disease. ${ }^{7}$ Therefore, elucidating the underlying molecular mechanism and finding novel diagnostic biomarkers and effective therapeutics for patients with GC are urgently needed.

LncRNAs are RNAs with more than 200 nucleotides in length but without protein-coding capacity. ${ }^{8}$ Numerous studies showed that dysregulation of IncRNA expression is associated with cellular processes and disease occurrence and development, such as tumor initiation. Functionally, lncRNAs can regulate a series of biological processes, including cell proliferation, apoptosis, autophagy, invasion, migration, epigenetic regulation, chromosome modification, and cell cycle. It also closely regulates tumor initiation, immune response, neurological diseases, and metabolic diseases. In terms of GC, many lncRNAs regulate $\mathrm{GC}$ progression at varying degrees; for instance, lncRNA AC093818.1 can promote GC metastasis. ${ }^{9}$ LncRNA UCA1 promotes the proliferation, migration, and immune escape and inhibits the apoptosis of GC cells. ${ }^{10}$ LncRNA LINC00963 is an oncogene that promotes the progression of cancer, including breast cancer, ovarian cancer, melanoma, and hepatocellular carcinoma. ${ }^{11-14}$ However, the function of LINC00963 in GC is still unknown.

DCs are antigen-presenting cells, and abnormal function of these cells causes immune-related diseases. For instance, DC-related immune tolerance promotes tumor development. $^{15}$ In tumor microenvironment, DCs are domesticated and then help tumor cells evade immunological surveillance. LncRNAs have been linked to the differentiation, maturation, and biofunctions of DCs. For instance, lnc-DC affects the differentiation and stimulating T-cell activation functions of DCs by regulating STAT3 transcription factor. ${ }^{16}$ However, the expression of LINC00963 and its function in DCs remain unclear.

In this study, we hypothesized that LINC00963 promotes GC progression. Then, a higher expression of LINC00963 was detected in GC tissues and cell lines. Knockdown of LINC00963 suppressed the proliferation, migration, invasion, and tumor growth of GC cells. LINC00963 promoted GC progression by acting as a competing endogenous RNA (ceRNA) to regulate the expression of miR-612 and CDC5L. Simultaneously, LINC00963 can also inhibit the function of DCs to accelerate tumor growth. This study might provide insights into the clinical diagnosis and treatment of GC by combining with DC-targeted immunotherapy.

\section{Materials and Methods Patients and Tissue Samples}

Sixty pairs of GC tissues and adjacent normal tissues were obtained from GC patients at the Suzhou Ninth People's Hospital. The written informed consents were obtained from patients. Tissue specimens were immediately frozen in liquid nitrogen and then stored at $-80^{\circ} \mathrm{C}$ for further study. This study was approved by the Ethics Committee of Suzhou Ninth People's Hospital.

\section{Cell Culture}

GC cell lines (AGS, BGC-823, SCG-7901 and HGC-27) and normal human gastric epithelium cell line GES-1 were purchased from the American Type Culture Collection (Manassas, VA, USA). Cells were cultured in Dulbecco's modified Eagle's medium (DMEM) (Invitrogen, Carlsbad, CA) with $10 \%$ fetal bovine serum (FBS, Invitrogen, Carlsbad, $\mathrm{CA}$ ) and $1 \%$ penicillin/streptomycin (Invitrogen, Carlsbad, CA) at $37^{\circ} \mathrm{C}$ in an atmosphere with $5 \% \mathrm{CO}_{2}$.

\section{Plasmid Construction and Cell Transfection}

To knockdown LINC00963 and CDC5L, small hairpin RNA (shRNA) and small interfering RNA (siRNA) specifically targeting LINC00963 (sh-LINC00963-1 and shLINC00963-2) and CDC5L (si-CDC5L-1 and si-CDC5L -2) were designed and purchased from GenePharma (Shanghai, China). The pcDNA vector and pcDNALINC00963 were synthesized and purchased from GenePharma (Shanghai, China). Plasmids and empty vector were transfected into GC cells with Lipofectamine 2000 (Invitrogen). The cells were harvested at 48 hours after transfection for following assays.

\section{Luciferase Reporter Assay}

The fluorescent reporter plasmids were constructed and purchased from Promega. In brief, complete fragments of LINC00963 and the 3'-UTR of CDC5L were amplified by PCR and cloned into downstream of the luciferase gene of pmirGLO dual-luciferase vector to generate LINC00963WT and CDC5L-WT plasmids. Correspondingly, LINC00963-MUT and CDC5L-MUT were constructed. Then, GC cells were transfected with LINC00963-WT, 
LINC00963-MUT fluorescent reporter plasmids, NC mimics or miR-612 mimics using Lipofectamine 2000, respectively. Similarly, GC cells were transfected with CDC5L-WT, CDC5L-MUT fluorescent reporter plasmids, NC mimics or miR-612 mimics using Lipofectamine 2000. Each well of 12 -well plate contained $0.5 \mu \mathrm{g}$ plasmids, 50 nmol mimics and $4 \mu \mathrm{L}$ Lipofectamine 2000 in $1 \mathrm{~mL}$ medium. Four replicate wells each group, and at least three experiments were conducted. After 48 hours, the luciferase activity was measured by the Dual-Luciferase Reporter Assay Kit (Promega) following manufacturer's protocol.

\section{Apoptosis Analysis}

The apoptosis of GC cells was analyzed by an Annexin $\mathrm{V}$ Apoptosis Detection Kit (BD, Biosciences). Briefly, after 48 hours of transfection, cells were harvested and resuspended by $500 \mu \mathrm{L} 1 \times$ binding buffer, then stained with $5 \mu \mathrm{L}$ Annexin V-FITC and $5 \mu \mathrm{L}$ PI for 15 minutes. Cells apoptosis rate was detected using a flow cytometry FACS (BD, Biosciences, USA).

\section{RNA Extraction and Quantitative Real-Time Polymerase Chain Reaction (qRT-PCR)}

Total RNA was extracted from GC tissues or cells using TRIzol reagent (Invitrogen). Then, RNA was reversed to cDNA by a M-MLV Reverse Transcriptase kit (Takara, Dalian, China). The SYBR Green PCR Kit (Takara) was used to detect the relative expression levels of genes on ABI 7500 Real-time PCR System (Applied Biosystems). GAPDH or U6 was used as endogenous control for mRNA or miRNA, respectively. The relative expression levels of genes were calculated by $2^{-\Delta \Delta \mathrm{Ct}}$ method.

\section{Western Blot}

Total protein was extracted from GC tissues or cells using radioimmunoprecipitation lysis buffer (Beyotime, Beijing, China). Protein concentrations were detected by the BCA Protein Assay Kit (Beyotime, Tiangen, China). Equal amounts of protein samples were separated by $8 \%$ or $10 \%$ sodium dodecyl sulfate-polyacrylamide gel electrophoresis and then transferred to Polyvinylidene Fluoride membrane (PVDF, Millipore, Germany). The membrane was blocked with fat-free milk (5\%) for 2 hours at room temperature and incubated with primary antibodies overnight at $4^{\circ} \mathrm{C}$. Then, the membranes were incubated with secondary antibodies for 1 hour at room temperature. The protein bands were detected by an enhanced chemiluminescence system (Pierce, Waltham, MA). In this study, primary antibodies included Vimentin (1:1000, Abcam), E-cadherin (1:1000, Abcam), Bcl2 (1:1000, Cell Signaling Technology), Bax (1:1000, Cell Signaling Technology), GAPDH (1:5000, Cell Signaling Technology) and CDC5L (1:1000, Cell Signaling Technology). Secondary antibodies were anti-mouse (1:2000, Cell Signaling Technology) and anti-rabbit (1:2000, Cell Signaling Technology).

\section{Transwell Assay}

Transwell assays were performed to detect the migration and invasion capacities of GC cells. For cell migration assay, a total of $5 \times 10^{4}$ AGS or BGC-823 cells were cultured in serum-free media and plated in the top chamber of 24-well plate-sized transwell inserts. The lower chambers contained medium with $10 \%$ FBS. After incubation for 24 hours, cells on the lower membrane surface were fixed with $4 \%$ paraformaldehyde and stained using $0.5 \%$ crystal violet. The migrated cells were counted with a microscope (Olympus) at least five random fields. For cell invasion assay, the transwell chambers treated with Matrigel (BD Biosciences), other steps were performed according to the protocol of migration assay.

\section{Cell Proliferation Assay}

Cell proliferation ability was examined using a Cell Counting Kit-8 (CCK-8) (Dojindo, Kumamoto, Japan). AGS or BGC-823 cells were seeded into 96-well plates $\left(2 \times 10^{3}\right.$ cells/well). Ten microliters of CCK-8 solution added into each well, cells were cultured at $37^{\circ} \mathrm{C}$ for 2 hours. Absorbance values were detected at $450 \mathrm{~nm}$ using a microplate reader and proliferation ability was calculated following the manufacturer's protocol.

\section{Xenograft Tumor Model}

BALB/c nude mice (female, 4 weeks of age) were purchased from the Chinese Academy of Medical Sciences. AGS cells $\left(2 \times 10^{6}\right.$ in $100 \mu \mathrm{L}$ PBS $)$ transfected with shRNA or sh-LINC00963 were injected subcutaneously into the underarm of mice $(n=6)$. After tumor formation, tumor size was measured every 4 days. Tumor volume was calculated using the formula: length $\times$ width $^{2} \times 1 / 2$. At 4 weeks after injection, all mice were euthanized and tumors were harvested for following assays. Animal studies were approved by the Animal Ethics Committee of 
Suzhou Ninth People's Hospital in accordance with guidelines of the US NIH.

\section{Bone Marrow-Derived Dendritic Cells (BMDCs) and Detection of DC}

\section{Maturation}

C57BL/6 (C57, 8 weeks old) mice were euthanized and BMDCs sorted from hind leg bones of mice. To generate DCs from BMDCs, BMDCs cells cultured in RPMI1640 medium with GM-CSF $(20 \mathrm{ng} / \mathrm{mL})$ and IL-4 $(10 \mathrm{ng} / \mathrm{mL})$ for 5 days at $37^{\circ} \mathrm{C}$ in an atmosphere with $5 \% \mathrm{CO}_{2}$. Then, BMDCs transfected with sh-NC or sh-LINC00963. Half of the medium were replaced by fresh media at 2 days. Next, BMDCs were harvested and planted in six-well plates, and treated with lipopolysaccharide (LPS, $200 \mathrm{ng} / \mathrm{mL}$ ) for 12 hours. After incubation, supernatants were collected for ELISA assay. DCs were analyzed by qRT-PCR or flow cytometry. For flow analysis, DCs were stained with antiCD11c-FITC, anti-MHC II-Percp (eBioscience, USA). The maturation of DCs was analyzed by flow cytometry FACS (BD, Biosciences, USA). Data were analyzed using FlowJo software (Ashland, OR, USA).

\section{ELISA}

The cytokines secreted from DCs were measured by ELISA kit (BD, Biosciences, CA, USA). As mentioned above, DCs supernatants were collected after treatment of LPS. The expression levels of IFN $\gamma$, TNF- $\alpha$, IL-6, IL-10, IL-12 were detected according to the manufacturer's instructions.

\section{Statistical Analysis}

All experiments were performed at least three replicates. Data were presented as the mean $\pm \mathrm{SD}$ and analyzed by GraphPad Prism 5.0 and SPSS 20.0 software. Comparisons between two groups or multiple groups were performed using Student's $\mathrm{s} t$-tests and one-way analysis of variance (ANOVA). $\mathrm{P}<0.05$ was considered statistically significant.

\section{Results}

\section{Overexpression of LINC00963 in GC} Tissues and Cell Lines

To reveal the effect of LINC00963 on GC progression, we first measured the expression level of LINC00963 in GC tissues and adjacent tissues by using qRT-PCR. Results showed that the expression of LINC00963 was significantly higher in GC tissues than in adjacent normal tissues $(\mathrm{n}=60)$ (Figure 1A). The characteristics of the patients with GC are shown in Table 1. Meanwhile, the expression of LINC00963 in GC cell lines and gastric epithelial cell line was detected. LINC00963 expression was upregulated in the GC cell lines but not in the gastric epithelial cell line (Figure 1B). Kaplan-Meier analysis showed the poor overall survival of GC patients with high LINC00963 expression (Figure 1C). Furthermore, LINC00963 overexpression promoted the lymph node metastasis and distant metastasis of GC (Figure 1D and E). The expression of LINC00963 was significantly increased in GC patients with different clinicopathological stages (Figure 1F). The main biological functions of LINC00963 were studied using LnCeVar (http://www.bio-bigdata.net/ $\underline{\mathrm{LnCeVar}}$ ) (Figure 1G). These results demonstrated that LINC00963 was overexpressed in GC tissues and cell lines.

\section{LINC00963 Affects the Proliferation, Apoptosis, Migration, and Invasion of GC Cells}

We investigated the effects of LINC00963 on the proliferation, apoptosis, migration, and invasion of GC cells in vitro. We first detected the efficiency of silencing or overexpressing LINC00963 in AGS and BGC-823 cells. Results indicated that LINC00963 expression was greatly suppressed in AGS and AGS cells transfected with shLINC00963-1 or sh-LINC00963-2 (Figure 2A). shLINC00963-2 was selected for follow-up experiments. LINC00963 overexpression was also achieved in the GC cells transfected with the LINC00963 overexpression plasmid (Figure 2B). CCK-8 assay demonstrated that proliferation was significantly higher or lower in the AGS and BGC-823 cells transfected with LINC00963 or shLINC00963-2/sh-LINC00963-1 compared with that in the control group, respectively (Figure $2 \mathrm{C}$ and $\mathrm{D}$, and Supplement Figure 1a, $\underline{b}$ ). Transwell assay was performed to prove that the migration and invasion capacities of AGS and BGC-823 cells were affected by LINC00963. Results showed that the migration and invasion capacities were markedly impeded in the LINC00963-silenced GC cells but were notably promoted in the LINC00963overexpressing GC cells (Figure 2E and F, and Supplement Figure 1c, d). Epithelial-mesenchymal transition (EMT) and apoptosis are key processes in cancer progression. In the present study, Bcl2 and EMT marker vimentin were suppressed while apoptosis-promoting 
A

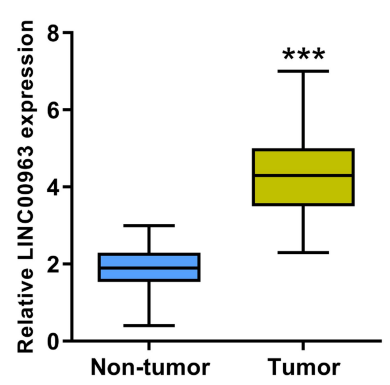

D

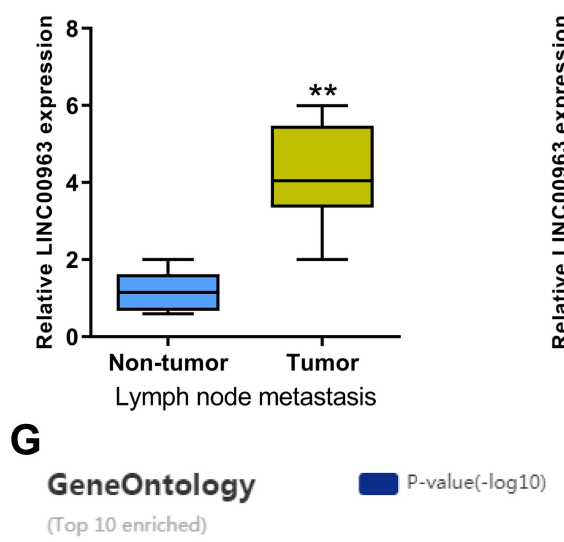

(Top 10 enriched)

RECEPTOR_SIGNALING_PROTEIN_SERINE_THREONINE_KINASE_ACTIVITY TRANSMEMBRANE_RECEPTOR_PROTEIN_KINASE_ACTIVITY AMYLOID_PRECURSOR_PROTEIN_METABOLIC_PROCESS RECEPTOR_SIGNALING_PROTEIN_ACTIVITY PROTEIN_MATURATION GLYCOPROTEIN_CATABOLIC_PROCESS NOTCH_SIGNALING_PATHWAY MAP_KINASE_ACTIVITY

SMAD_BINDING REGULATION_OF_TRANSCRIPTION
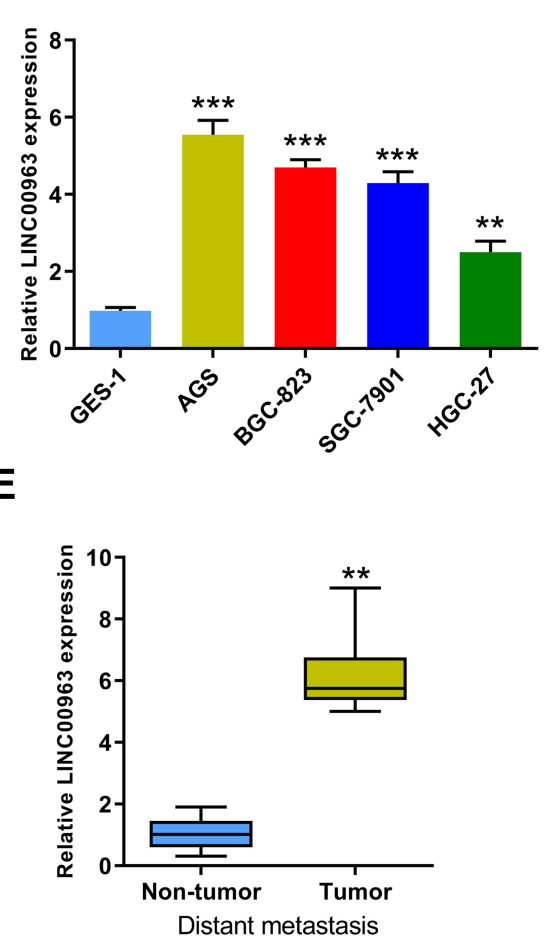

C

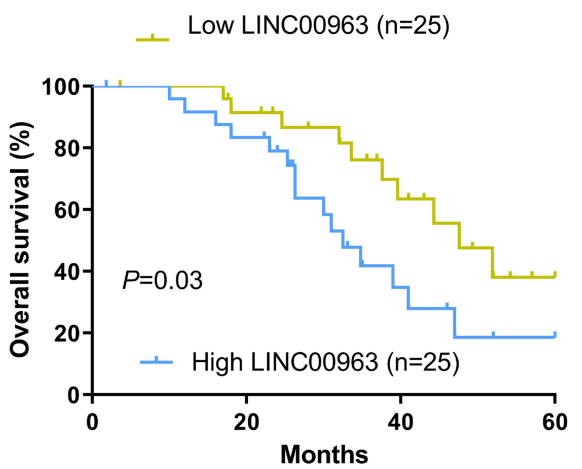

$\mathbf{F}$

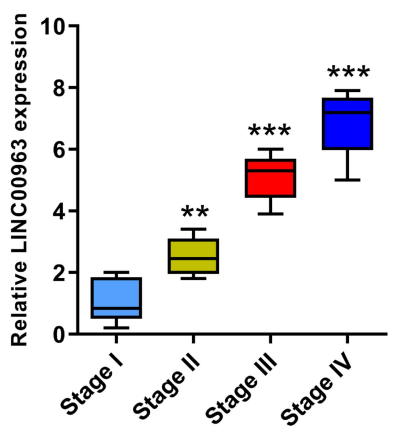

Functional Pathways P-value(- $\log 10)$

(Top 10 enriched)
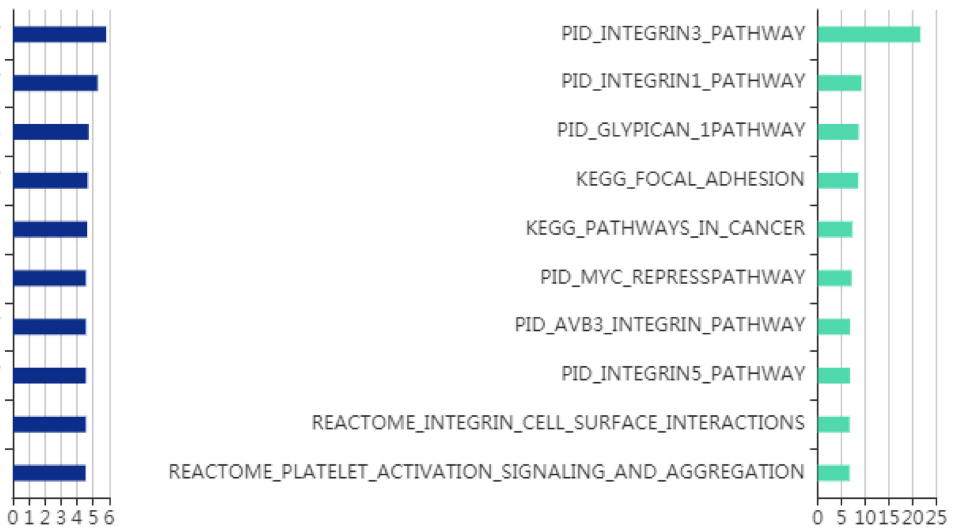

Figure I Over-expression of LINC00963 in GC tissues and cell lines. (A) The LINC00963 expression was detected in GC tissues and adjacent non-tumor tissues by qRTPCR, $(n=60)$. (B) Relative expression levels of LINC00963 were detected by qRT-PCR in GC cell lines (AGS, BGC-823, SGC-790I and HGC-27) and normal gastric epithelial cell line (GES-I). (C) Kaplan-Meier survival curve of GC patients with high or low expression of LINC00963. (D and E) The LINC00963 expression was detected in GC patients with/without lymph node metastasis or distant metastasis by qRT-PCR. (F) Relative expression levels of LINC00963 in GC patients at different clinical stages.

(G) Functional enrichment analysis of LINC00963 was performed by online tools LnCeVar (http://www.bio-bigdata.net/LnCeVar/). **P < $0.0 \mathrm{I}$ and ***P < $0.00 \mathrm{I}$.

related proteins Bim, Bax, and EMT marker E-cadherin were significantly enhanced in the LINC00963-silenced GC cells (Figure 2G and H, and Supplement Figure 1f). The opposite effects were found in GC cells with high LINC00963 expression (Figure 2G and H). In addition, LINC00963 knockdown significantly promoted the apoptosis of GC cells (Figure 2I and Supplement Figure 1e). These results indicate that silencing LINC00963 can accelerate the apoptosis and inhibit the proliferation, migration, and invasion of GC cells.

\section{LINC00963 Functions as a ceRNA to Bind miR-6I 2}

Increasing studies have suggested that lncRNAs regulate the progression of various cancers, including GC, by 
Table I Relationship Between LINC00963 and Clinicopathological Features of Patients with GC $(n=60)$

\begin{tabular}{|c|c|c|c|c|}
\hline \multirow[t]{2}{*}{ Characteristics } & \multirow[t]{2}{*}{$\mathbf{n}$} & \multicolumn{2}{|c|}{$\begin{array}{l}\text { LINC00963 } \\
\text { Expression }\end{array}$} & \multirow[t]{2}{*}{$P$ value } \\
\hline & & $\begin{array}{l}\text { Low } \\
(n=28)\end{array}$ & $\begin{array}{l}\text { high } \\
(n=32)\end{array}$ & \\
\hline \multicolumn{5}{|l|}{ Age } \\
\hline$<60$ & 29 & 14 & 15 & \\
\hline$\geq 60$ & 31 & 14 & 17 & 0.809 \\
\hline \multicolumn{5}{|l|}{ Gender } \\
\hline Female & 31 & 13 & 18 & \\
\hline Male & 29 & 15 & 14 & 0.448 \\
\hline \multicolumn{5}{|l|}{ Tumor size } \\
\hline$\leq 5 \mathrm{~cm}$ & 34 & 22 & 12 & \\
\hline$>5 \mathrm{~cm}$ & 26 & 6 & 20 & $0.001 *$ \\
\hline \multicolumn{5}{|l|}{ Lymph node metastasis } \\
\hline No & 33 & 20 & 13 & \\
\hline Yes & 27 & 8 & 19 & $0.002 *$ \\
\hline \multicolumn{5}{|l|}{ Distant metastasis } \\
\hline No & 43 & 25 & 18 & \\
\hline Yes & 17 & 3 & 14 & $0.01 *$ \\
\hline \multicolumn{5}{|l|}{ TNM Stage } \\
\hline I/II & 26 & 17 & 9 & \\
\hline III/IV & 34 & 11 & 23 & $0.01 *$ \\
\hline
\end{tabular}

Notes: Low/high by the sample median. $\chi^{2}$ test. ${ }^{*} \mathrm{P}<0.05$ indicates statistical significance.

functioning as ceRNAs to regulate the expression of miRNAs. Thus, a potential target miR-612 of LINC00963 was predicted in DIANALncBase (Figure 3A). Subsequently, dual-luciferase reporter gene assay was performed. Results showed that luciferase activity decreased when miR-612 mimics were co-transfected with the LINC00963-WT (00963-WT) plasmid but did not significantly change in the LINC00963-MUT (00963-MUT) group (Figure 3B). Consistently, miR-612 inhibitors increased luciferase activity (Figure 3C). Pearson's correlation analysis revealed a negative correlation between miR612 expression and LINC00963 (Figure 3D). Furthermore, miR-612 expression was downregulated in GC tissues and cell lines (Figure $3 \mathrm{E}$ and $\mathrm{F}$ ). The interaction between LINC00963 and miR-612 in GC cells was further studied. The expression of miR-612 was upregulated in AGS and BGC-823 cells with low LINC00963 level. High LINC00963 expression suppressed miR-612 expression in GC cells (Figure 3G). Similarly, LINC00963 expression was negatively regulated by miR-612 (Figure $3 \mathrm{H}$ ). In addition, miR-612 overexpression inhibited the proliferation, migration, invasion, and EMT and promoted the apoptosis of GC cells, but they were rescued by LINC00963 overexpression (Figure 3I-N). These results illustrated that the suppressor effect of miR-612 on GC progression was abolished by LINC00963.

\section{LINC00963 Enhances CDC5L Expression by Targeting miR-6I2 in GC Cells}

A total of 122 candidate targets of miR- 612 were obtained by using mRNA target prediction software miRWalk, TargetScan, and miRDB (Figure 4A). Considering the function of CDC5L in cell division cycle and the previous reports about CDC5L in cancer, we selected CDC5L for further research and presented the binding sites of miR-612 and CDC5L (Figure 4B). Dual-luciferase assay showed that luciferase activity was decreased in the cells co-transfected with miR-612 mimics and CDC5L-WT plasmids (Figure 4C) but was evidently increased in the cells co-transfected with miR612 inhibitors and CDC5L-WT plasmids (Figure 4D). In addition, the function of CDC5L in GC was explored. Data in Oncomine databases showed that the mRNA expression levels of CDC5L are elevated in different human cancers, including breast cancer, cervical cancer, head and neck cancer, and leukemia (Figure 4E). Further analysis showed that the mRNA expression of CDC5L was upregulated in $\mathrm{GC}$ in various datasets in the Oncomine database (Figure 4F-J). In addition, $\mathrm{CDC} 5 \mathrm{~L}$ was overexpressed in various $\mathrm{GC}$ datasets, and a meta-analysis was performed (Figure 4K). Furthermore, the DNA alteration frequency of CDC5L was confirmed in the cBioportal database to study the alterations of CDC5L in different cancers (Figure 5A). The gene alteration of $\mathrm{CDC} 5 \mathrm{~L}$ was $21 \%$ in patients with $\mathrm{GC}$ in the cBioportal database (Figure 5B and C). The expression of CDC5L was also detected in GC tissues and cell lines by using qRT-PCR. Results demonstrated that CDC5L expression was higher in GC tissues and cell lines than in the control group (Figure 6A and B). In addition, CDC5L expression was positively or negatively regulated by LINC00963 or miR-612 in GC cells (Figure 6C and D). The knockdown efficiency of CDC5L in GC cells was evaluated (Figure 6E), and si-CDC5L-1 was selected for followup experiments. Whether or not LINC00963 promotes GC progression by mediating CDC5L expression was investigated. Results showed that the migration and invasion abilities of GC cells were significantly attenuated after transfection with si-CDC5L, whereas LINC00963 
A

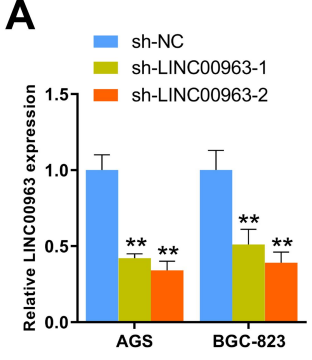

B

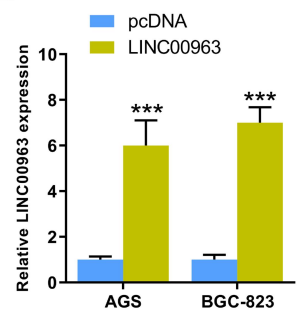

C

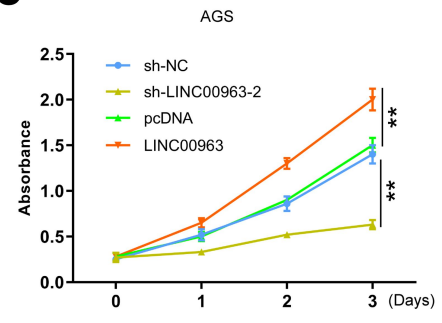

D

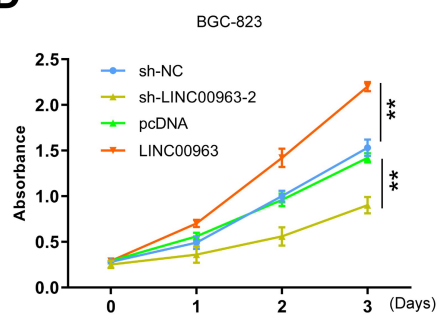

E

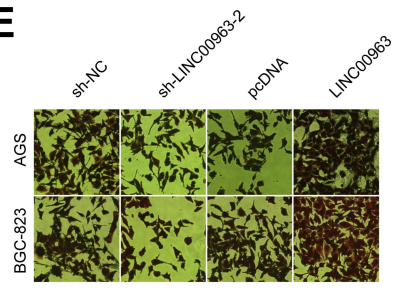

Migration

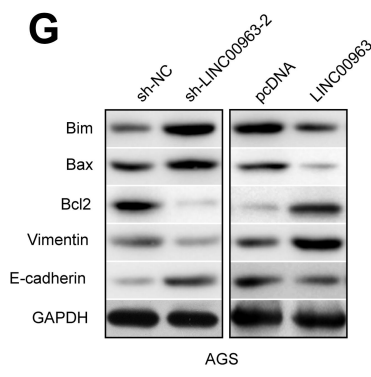

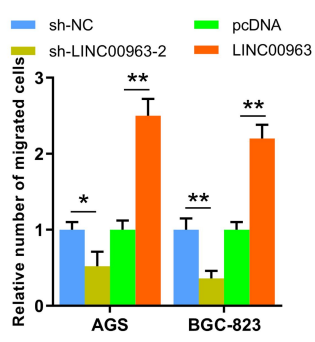

$\begin{array}{ll}\text { sh-NC } & \text { pcDNA } \\ \text { sh-LINC00963-2 } & =\text { LINC00963 }\end{array}$

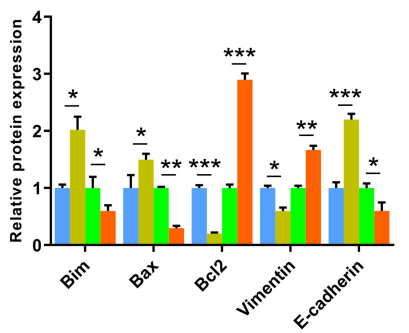

F

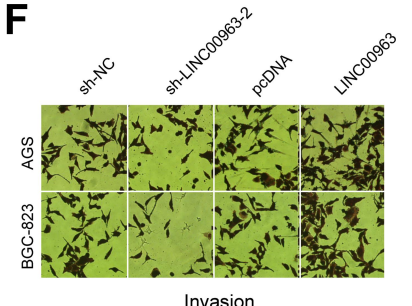

Invasion
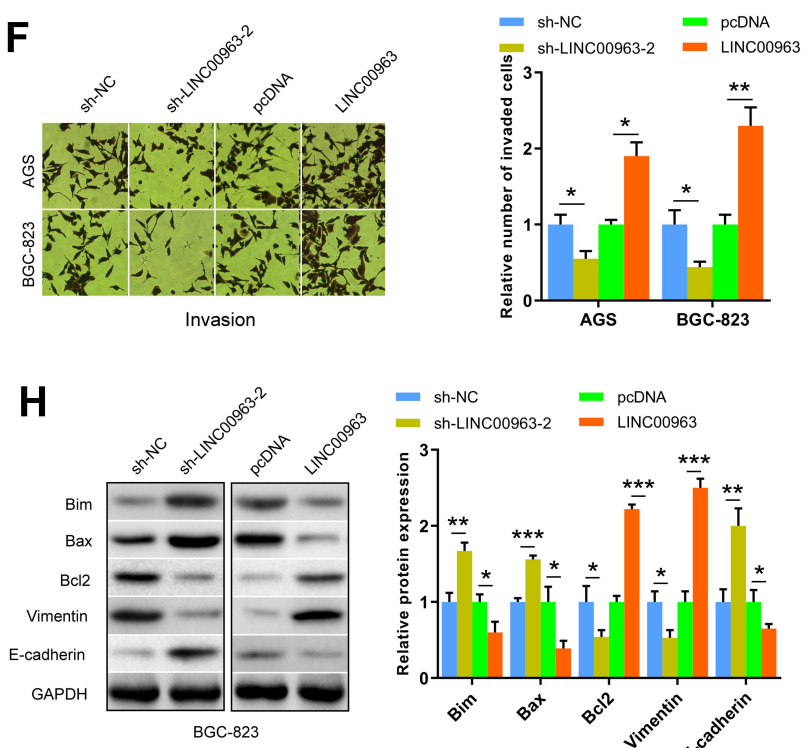

$\begin{array}{ll}\text { - sh-NC } & \text { pcDNA } \\ \text { sh-LINC00963-2 } & \text { LINC00963 }\end{array}$
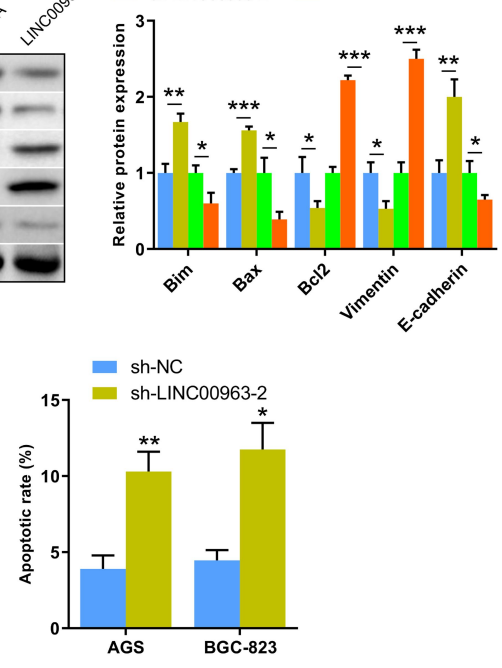

I
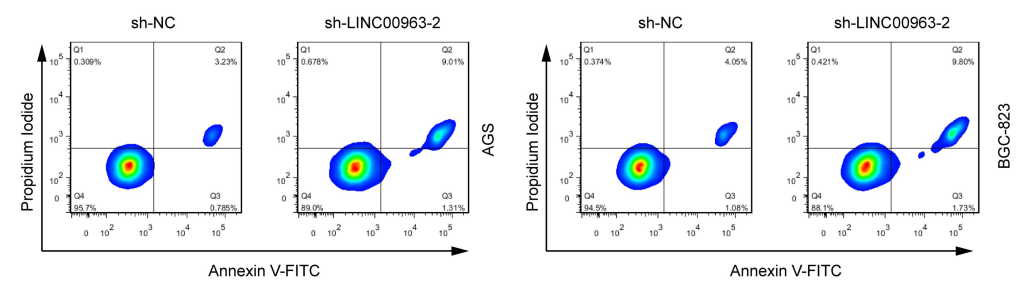

Annexin V-FITC

Figure 2 LINC00963 affected the proliferation, apoptosis, migration and invasion of GC cells. (A and B) The knockdown and overexpression of LINC00963 were confirmed in AGS and BGC-823 cells by qRT-PCR. (C and D) The proliferation ability of AGS and BGC-823 cells was analyzed by CCK8 assays. (E and F) Migration and invasion capacities of AGS and BGC-823 cells were evaluated by transwell assays. (G and $\mathbf{H}$ ) The expression levels of EMT-related (Vimentin and E-cadherin) and apoptosisrelated (Bim, Bax and Bcl2) proteins were analyzed by Western blot in AGS and BGC-823 cells. (I) Flow cytometry was used to detect the apoptosis rate of AGS and BGC823 cells transfected with sh-LINC00963-2. $* \mathrm{P}<0.05$, **P $<0.01$ and $* * * \mathrm{P}<0.001$.

overexpression rescued the suppression effect (Figure 6F and G). Moreover, the effects of CDC5L on the apoptosis and EMT of GC cells were studied. Flow cytometry and Western blot indicated that CDC5L knockdown obviously promoted cell apoptosis and inhibited EMT, but these effects were markedly reversed by LINC00963 overexpression (Figure 6H and I). The positive correlation between LINC00963 and CDC5L expression was confirmed in GC tissues (Figure 6J). The roles of miR-612 in CDC5L protein expression were investigated using Western blot. Results showed that the protein expression of CDC5L was significantly suppressed in the GC cells transfected with miR-612 mimics but was promoted in the GC cells transfected with miR-612 inhibitors (Figure 6K). Last, we observed in cBioPortal that the overall survival rate of GC patients with altered CDC5L slightly decreased, but the difference was not statistically significant (Figure 6L). Kaplan-Meier analysis showed the poor overall survival of GC patients with high CDC5L expression in our study (Supplement Figure 1g). In general, these results showed that LINC00963 regulated GC progression by directly targeting miR-612 to promote CDC5L expression. 
A

LNC00963-WT 5'...GAUUACAUGUGCGUGCCACCUUGCCCAGC....3'

hsa-miR-612 3 '...CUCGUCUUCCGAGGGGACGGGUCG...5 LNC00963-MUT 5'... GAUUACAUGUGCGUGCCACGGACGGGUCC... 3'

\section{E}

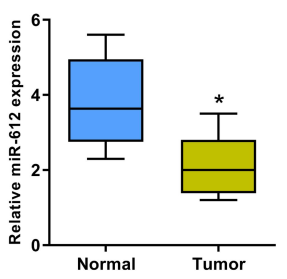

I

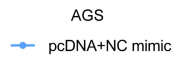

-

$\left.{ }^{2.0}\right]=$ pcDNA+miR-612 mimic

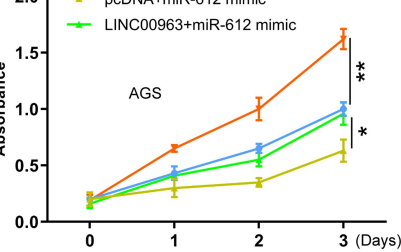

F

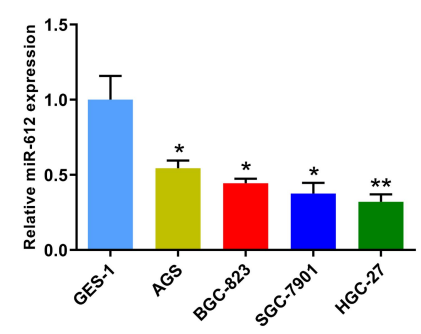

J

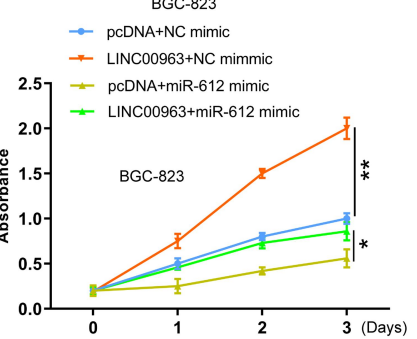

C

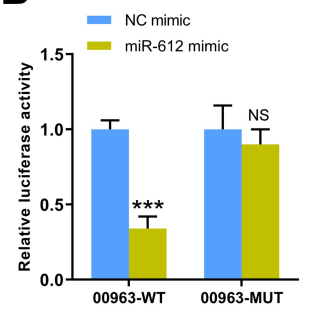

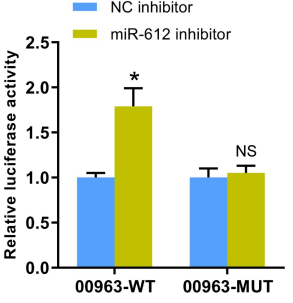

G

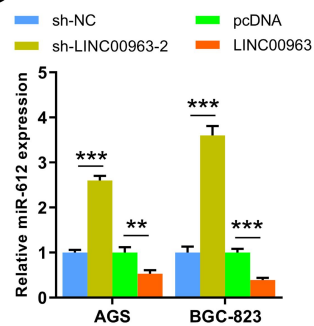

K

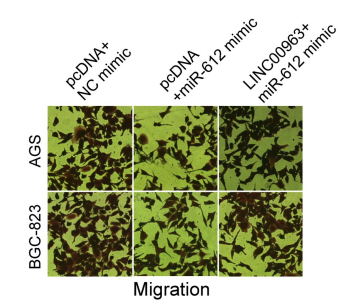

D

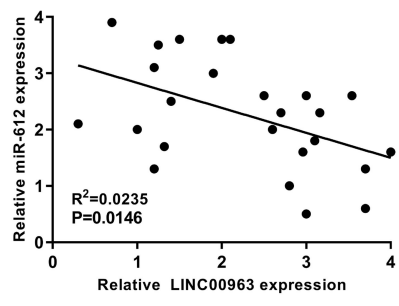

H

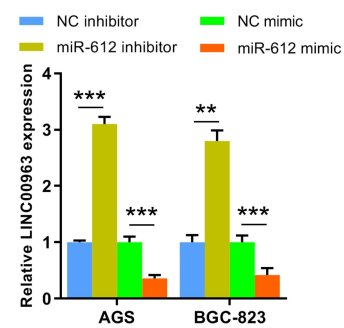

$=\underset{\text { NC mimic }}{\text { pcDNA+ }}=\underset{+m i R-612 \text { mimic }}{\text { pcDNA }}$
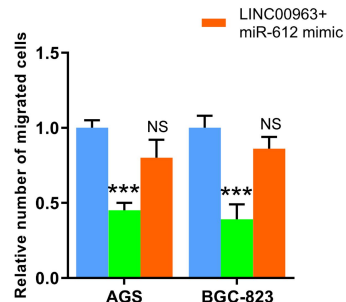

$\mathbf{L}$
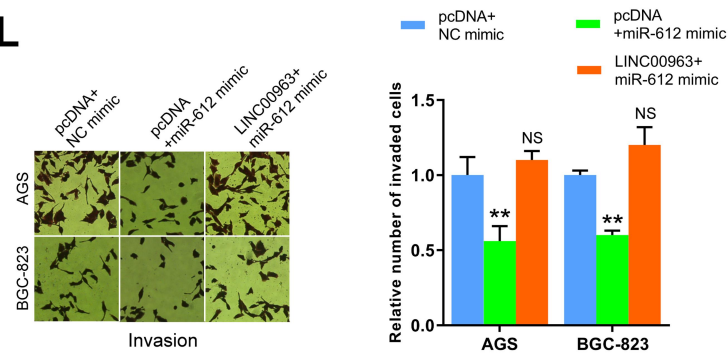

N

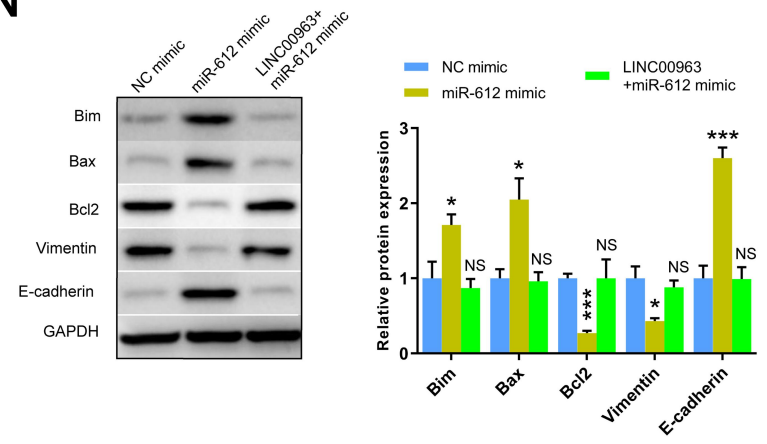

M

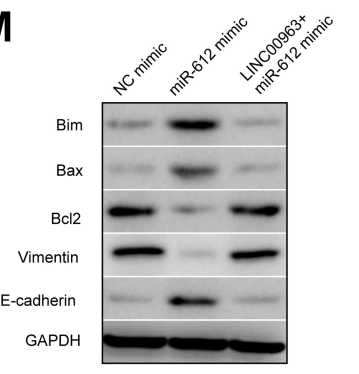

- NC mimic $\quad \begin{aligned} & \text { LINC00963 } \\ & + \text { miR-612 mimic }\end{aligned}$

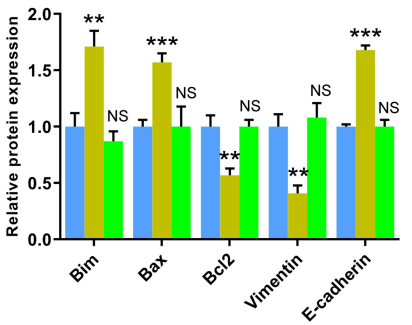

Figure 3 MiR-6I2 was a direct target of LINC00963. (A) Potential binding sequence between LINC00963 and miR-6I2 was predicted by Lncbase. (B and C) Dual-luciferase assays showed that the reduced or increased luciferase activities in AGS cells transfected with miR-6I2 mimics or inhibitors. (D) The negative relation between LINC00963 and miR-6I2 was analyzed in GC tissues. (E) Expression of miR-6I2 was detected in GC tissues and adjacent non-tumor tissues by qRT-PCR. (F) Relative expression levels of miR-6I2 in GC cell lines and normal gastric epithelial cell line. (G) The expression of miR-6I2 in AGS and BGC-823 cells with differential expression of LINC00963. (H) LINC00963 expression was detected in AGS and BGC-823 cells transfected with miR-6I2 mimics or inhibitors. (I-L) CCK8 and transwell assays of AGS and BGC-823 cells transfected with LINC00963 and/or miR-6I2 mimics. ( $\mathbf{M}$ and $\mathbf{N}$ ) The expression of EMT-related (Vimentin and E-cadherin) and apoptosis-related (Bim, Bax and Bcl2) proteins in AGS and BGC-823 cells transfected with LINC00963 and/or miR-6I2 mimics. *P $<0.05, * * \mathrm{P}<0.01$ and $* * * \mathrm{P}<0.001$.

Abbreviation: NS, not significant. 
A

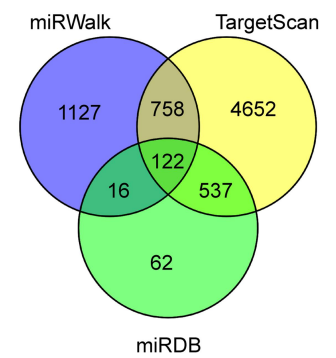

E

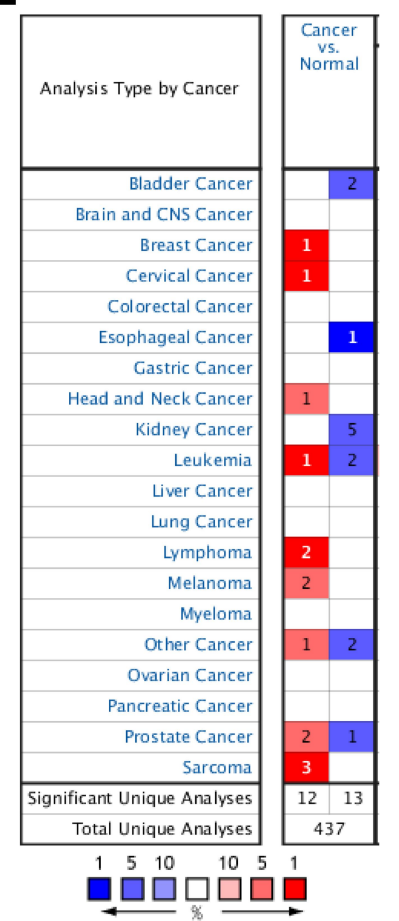

B

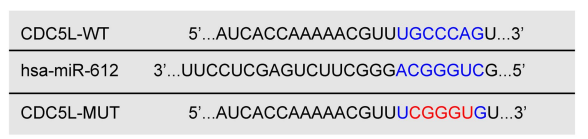

F

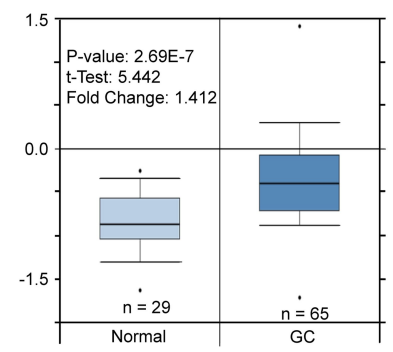

G

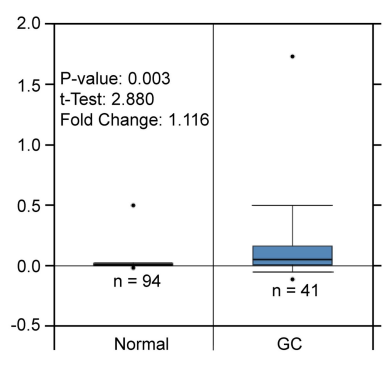

J
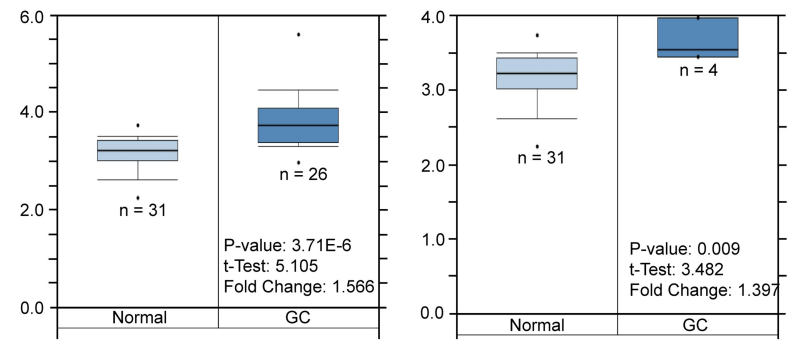

C

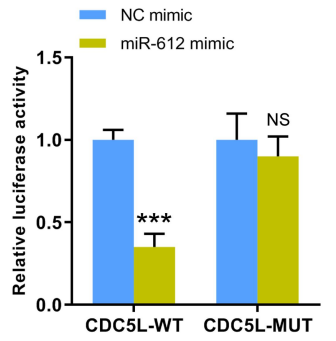

D

- NC inhibitor - miR-612 inhibitor

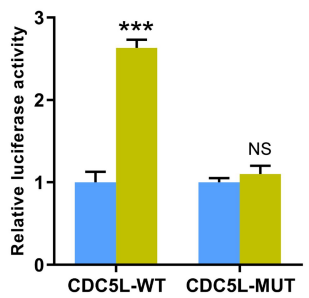

H

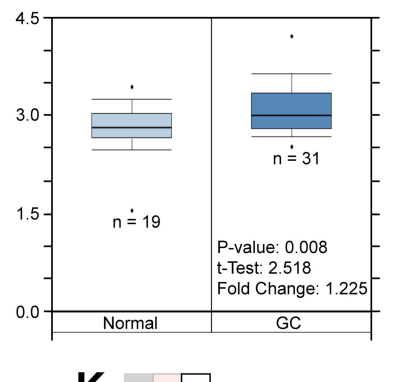

K

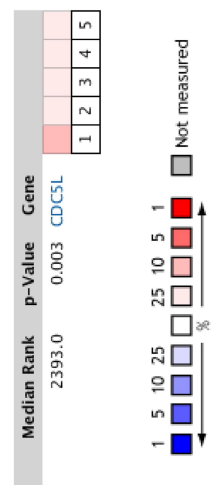

Figure 4 LINC00963 regulated CDC5L expression by targeting miR-6I2 in GC cells. (A) Potential targets of miR-6I2 were predicted by miRWalk, TargetScan and miRDB. (B) Binding sequence between CDC5L and miR-6I2. (C and D) Dual-luciferase reporter assays were used to confirm the binding between miR-6I2 and CDC5L. (E) CDC5L mRNA expression in different studies in Oncomine database. (F-J) The CDC5L mRNA expression was analyzed in human GC tissues and gastric normal tissues by Oncomine database (F Chen gastric. G TCGA gastric. $\mathbf{H}$ Cho gastric. I and J DErrico gastric). (K) Meta-analysis of CDC5L mRNA expression in five studies in Oncomine database (I, Chen gastric. 2, Cho gastric. 3, Deng gastric. 4, DErrico gastric. 5, TCGA gastric.). ***P $<0.00 \mathrm{I}$.

Abbreviation: NS, not significant.

\section{Knockdown of LINC00963 Restrained Tumor Growth of GC in vivo}

A xenograft mouse model of tumor was established to verify the role of LINC00963 in the tumor growth of GC in vivo. Results showed that tumor volume and weight significantly decreased in the group with low LINC00963 expression (Figure 7A-C). Moreover, the expression levels of LINC00963 and CDC5L were inhibited in the group with low LINC00963 expression (Figure 7D-F). LINC00963 knockdown increased the expression of miR612 (Figure 7G). Considering that DCs play a critical role in presenting tumor antigens and activating anti-tumor immunity response, we analyzed whether or not the proportion of DCs in the tumor changes in the different groups. Interestingly, results indicated that the proportion of DCs in tumor tissues significantly increased in the group with low LINC00963 expression (Figure 7H). These results demonstrated that inhibition of LINC00963 greatly suppressed tumor growth and increased the proportion of DCs in tumor in vivo.

\section{LINC00963 Inhibited Maturation of DCs by Targeting miR-6I 2 in vitro}

The expression levels of LINC00963 and miR-612 in the differentiation and maturation of DCs were measured to explore the potential role of LINC00963 on DC. qRT-PCR 
A

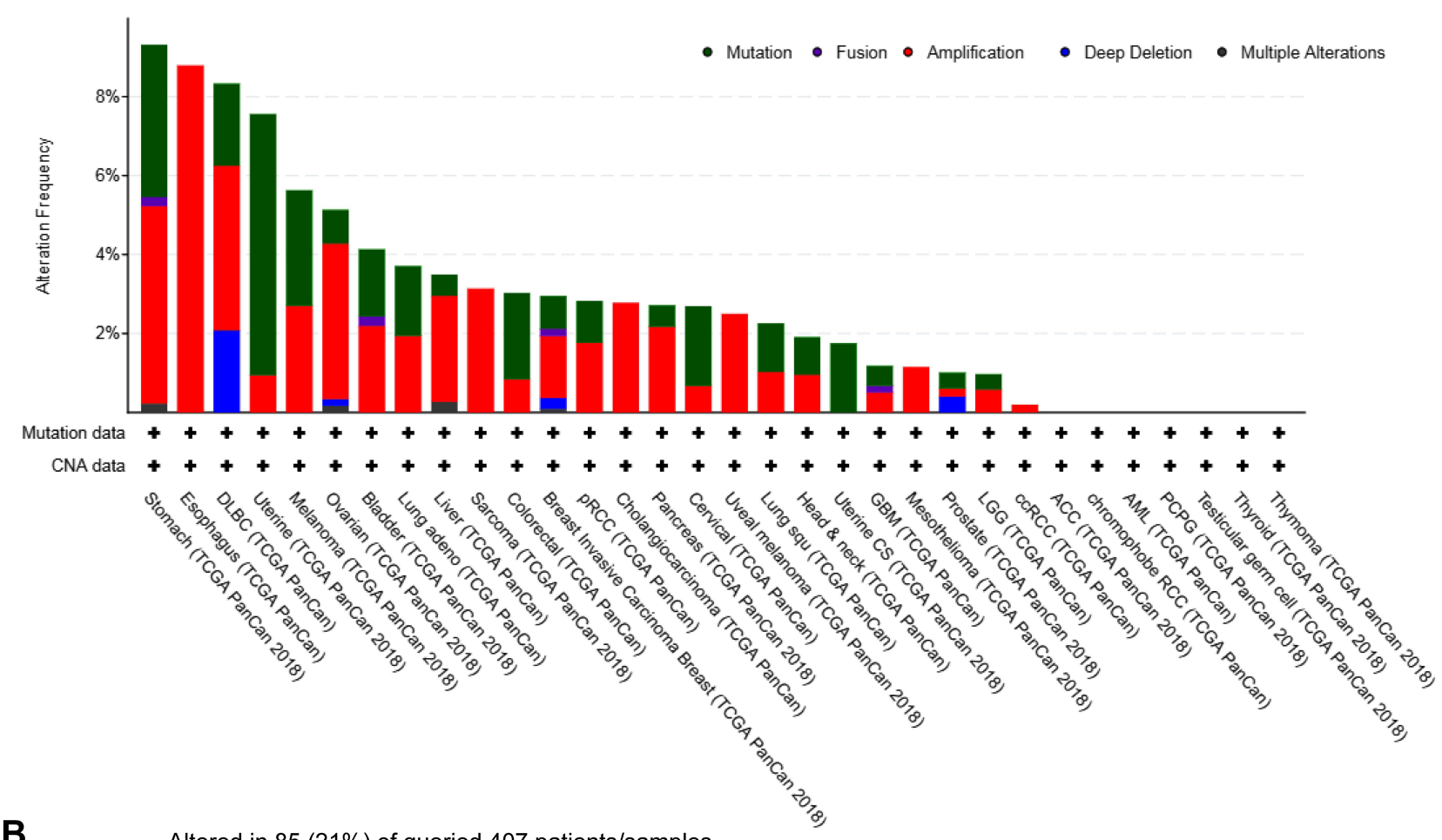

B Altered in $85(21 \%)$ of queried 407 patients/samples



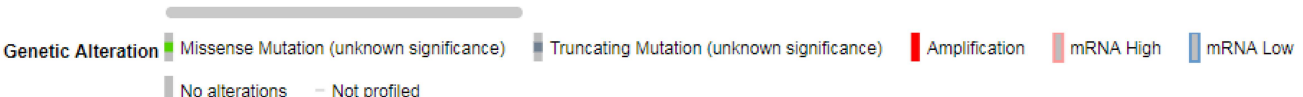

C

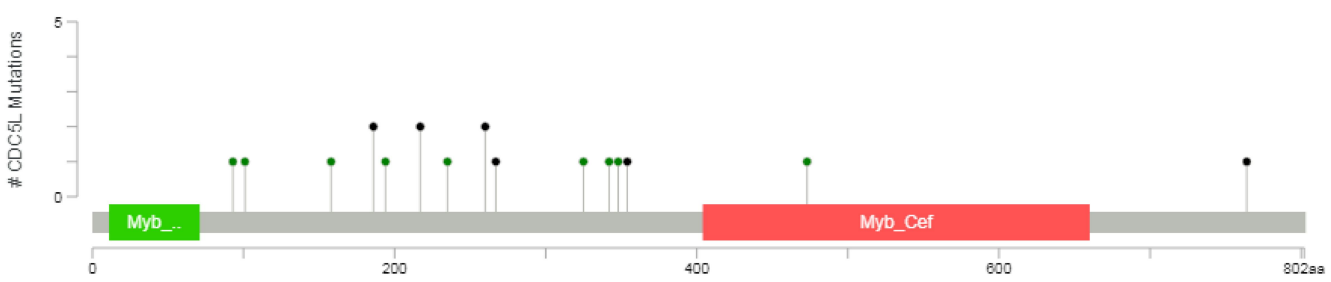

Figure 5 The mutation of CDC5L in human cancers. (A) Cross-cancer summary of CDC5L alterations frequencies from $\mathrm{cBioPortal}$. (B) The percentage of CDC5L alteration in stomach adenocarcinoma (TCGA, PanCancer Atlas). (C) The loci distribution of mutations in CDC5L from cBioPortal.

analysis revealed a negative correlation between LINC00963 and miR-612 in DCs (Figure 8A). DCs were transfected with sh-LINC00963, LINC00963, miR-612 mimics, and miR-612 inhibitors. Results of qRT-PCR showed that LINC00963 and miR-612 were successfully overexpressed or knocked down (Figure 8B and C). In addition, LINC00963 negatively regulated miR-612 expression in DCs (Figure 8D). During maturation, DCs express special surface markers and secretes cytokines. In the present study, qRT-PCR and ELISA analyses revealed that the levels of CD80, CD8, CD86, MHCII, TNF- $\alpha$, IFN$\gamma$, IL-6, and IL-10 increased and the level of IL-10 decreased in the DCs transfected with miR-612 mimics, but the overexpression of LINC00963 significantly reversed these results (Figure $8 \mathrm{E}$ and $\mathrm{F}$ ). The effects of LINC00963 and miR-612 on DC maturation were investigated using flow cytometry. Similar to the results in vivo, the proportion of DCs significantly increased in the group with low LINC00963 expression (Figure 8G). Moreover, the proportion of DCs transfected with miR-612 mimics increased, and this effect was rescued by LINC00963 (Figure $8 \mathrm{H}$ ). Finally, the flowchart of this study is presented in Figure 8I. These results indicated that LINC00963 inhibited the maturation of DCs by targeting miR-612. 

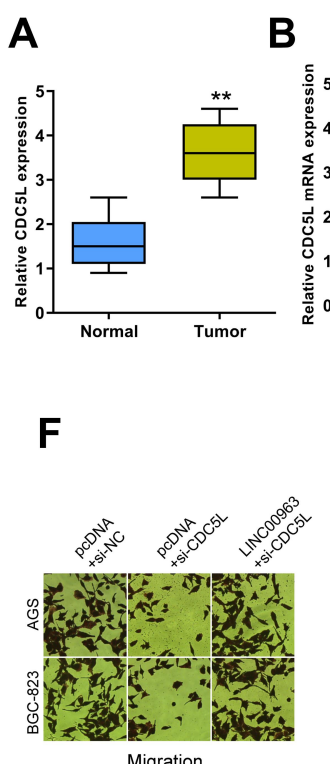

Migration
B

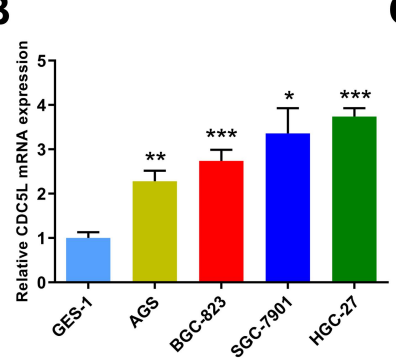

C

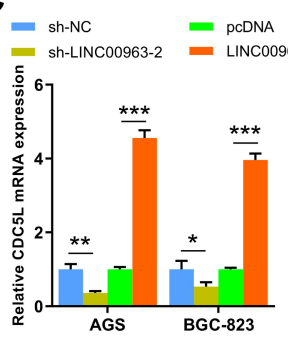

D

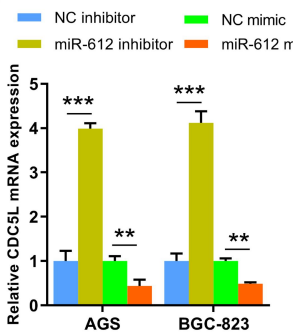

E

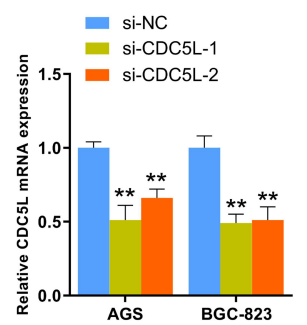

G
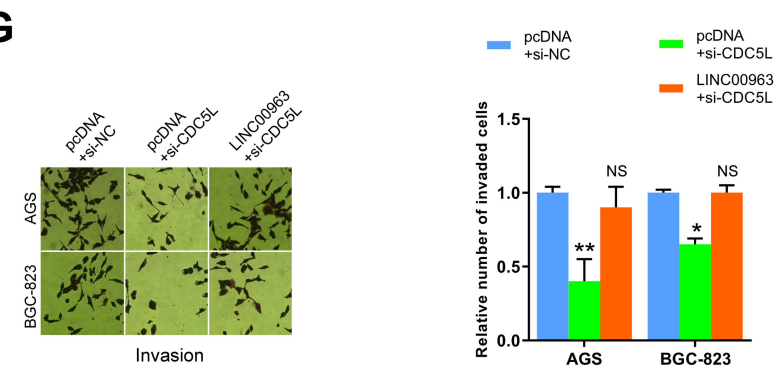

H
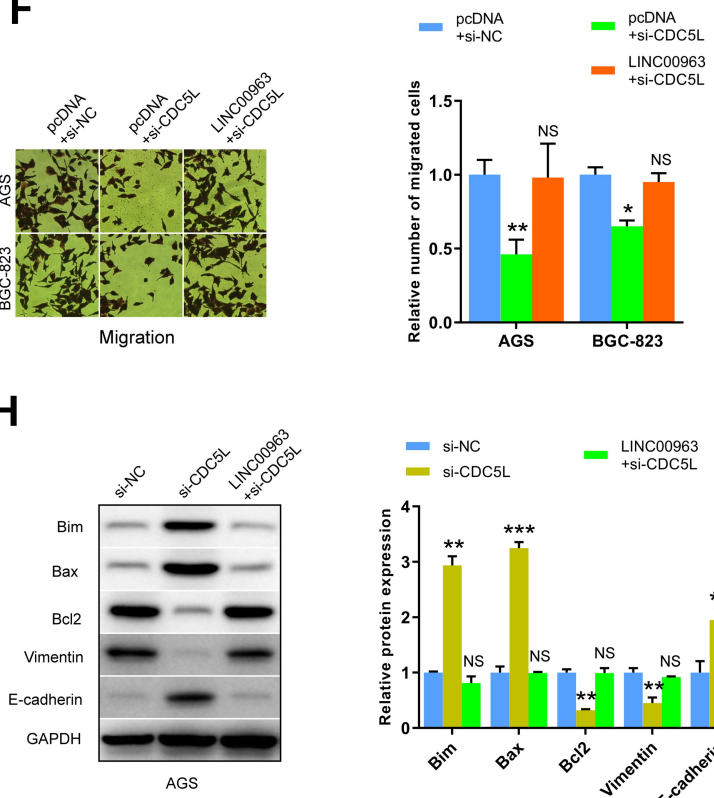

I
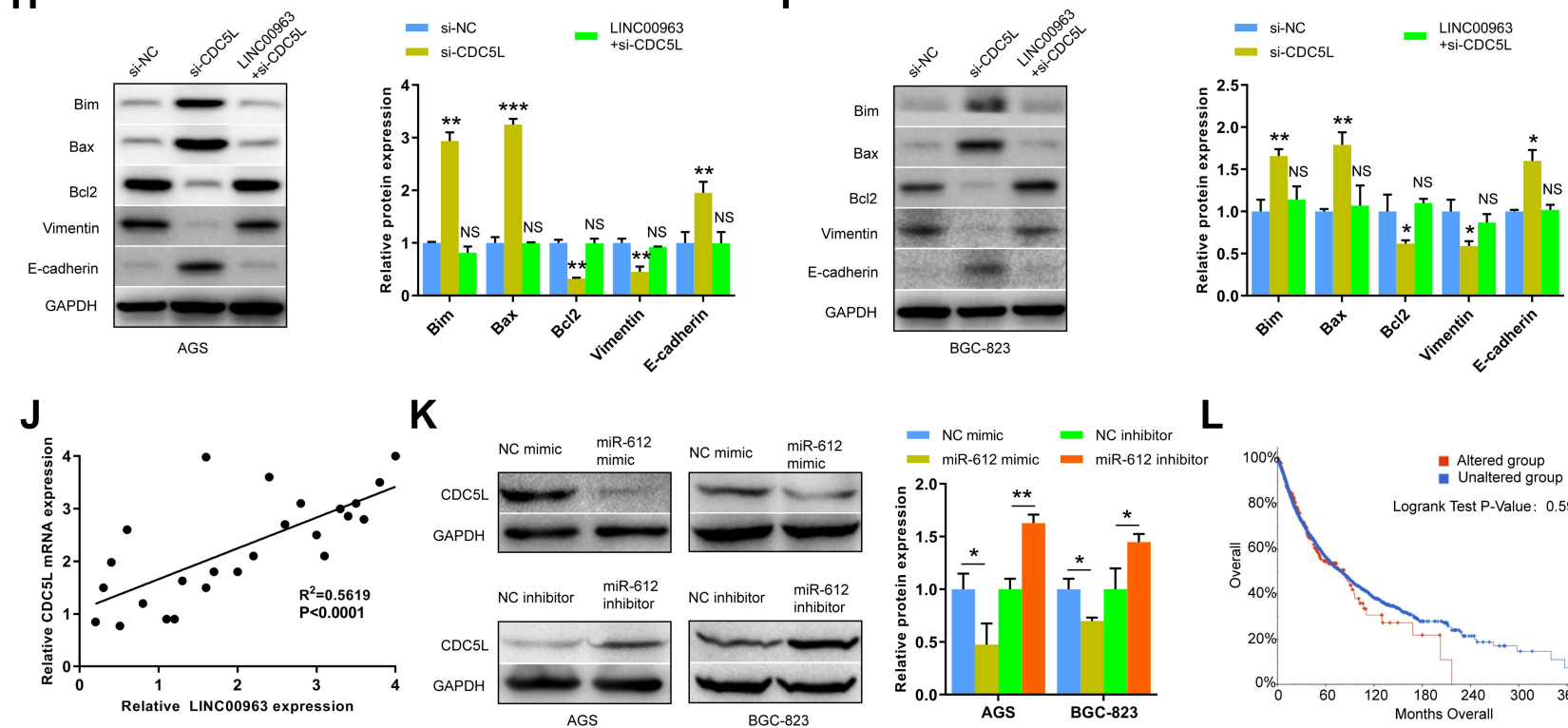

K
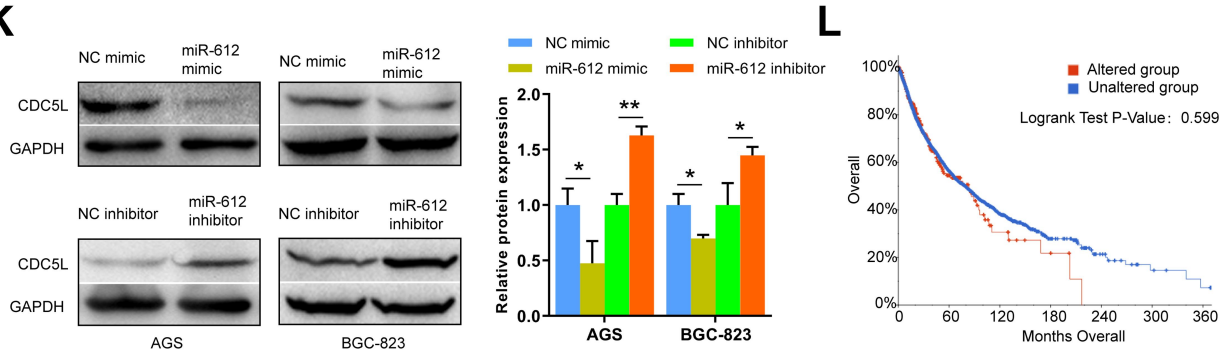

Figure 6 CDC5L affected the progression of GC in vitro. (A and B) Expression of CDC5L was significantly increased in GC tissues and cell lines. (C and D) The positive or negative relations between CDC5L and LINC00963 or miR-6I2 were confirmed by qRT-PCR. (E) The knockdown efficiency of CDC5L. (F and $\mathbf{G})$ Transwell assays of AGS and BGC-823 cells transfected with LINC00963 and/or si-CDC5L. (H and I) Expression of EMT-related (Vimentin and E-cadherin) and apoptosis-related (Bim, Bax and Bcl2) proteins in AGS and BGC-823 cells transfected with LINC00963 and/or miR-6I2 mimics. (J) Linear correlation analysis was performed between LINC00963 and miR-6I 2 in GC tissues. (K) The expression of CDC5L in protein level in AGS and BGC-823 cells transfected with miR-6I2 mimics or inhibitors. (L) The overall survival rate of GC patients with or without altered CDC5L from cBioportal database. $* \mathrm{P}<0.05$, **P $<0.0 \mathrm{I}$ and ***P $<0.00 \mathrm{I}$.

Abbreviation: NS, not significant.

\section{Discussion}

The occurrence and development of GC, as a common type of gastrointestinal cancer, involve a multi-factor process. Despite the continuous understanding of the pathogenesis of GC and the advancement in treatment methods, patients with GC still suffer from high mortality. ${ }^{17}$ Recent studies have found that IncRNAs are closely associated with the tumorigenesis of multiple cancers, including GC. ${ }^{18,19}$ For instance, IncRNA REG1CP promotes tumorigenesis through mediating REG3A transcription. ${ }^{20}$ LncNB1 promotes tumorigenesis by interacting with ribosomal protein RPL35. ${ }^{21}$ LncRNA NEAT1 impairs the DNA repair machinery and triggers anti-tumor activity in multiple myeloma. ${ }^{22}$ LncRNA p53-stabilizing and -activating RNA promotes p53 signaling and suppresses hepatocellular carcinoma. ${ }^{23}$ LINC00963 promotes breast cancer progression by targeting 
A

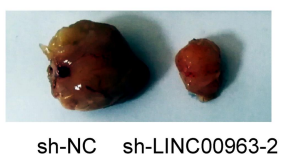

E

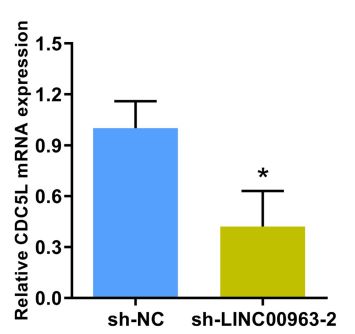

B

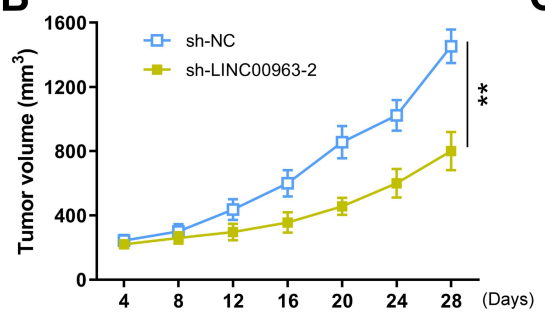

F
C
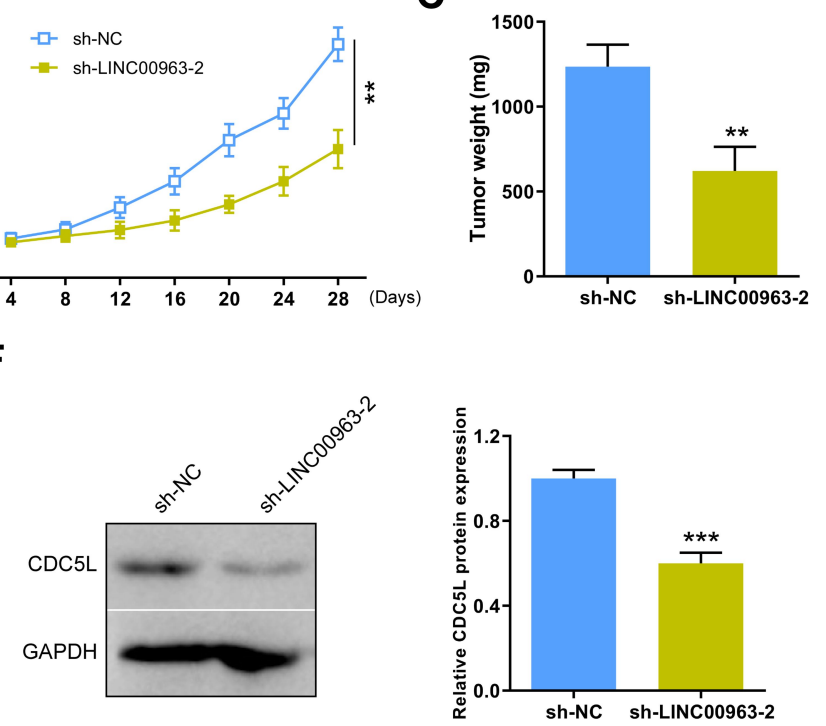

D

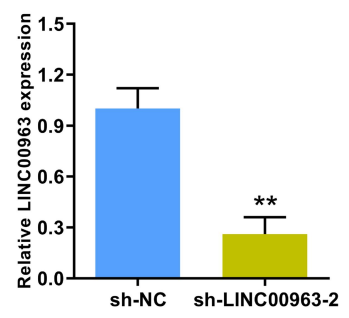

G

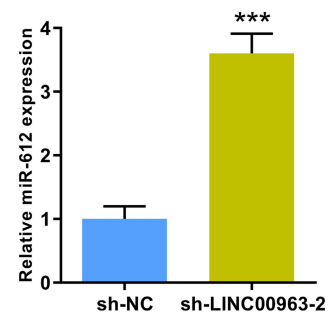

H
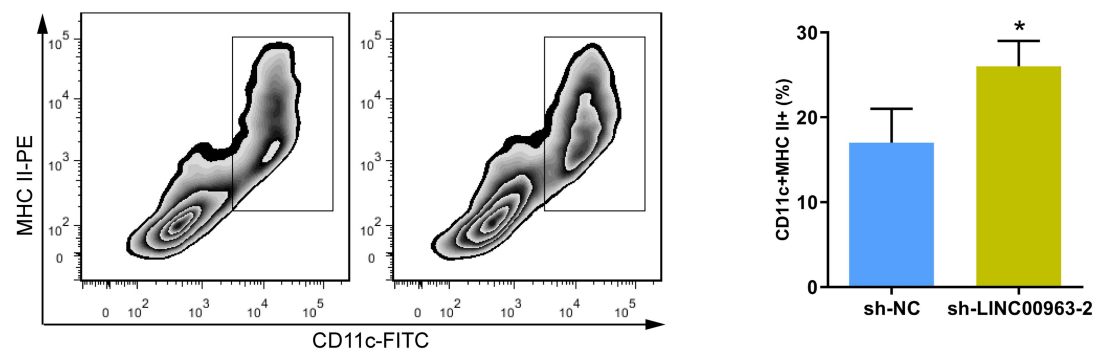

Figure 7 Knockdown of LINC00963 inhibited GC tumor growth in vivo. (A) Imaging of representative tumors from LINC00963 knockdown and its negative control group. (B) The tumor volume were measured at the scheduled time. (C) Tumor weight. (D and E) The expression levels of LINC00963 and CDC5L were analyzed in tumor tissues by qRT-PCR. (F) The expression of CDC5L in protein level in tumor tissues. (G) QRT-PCR analysis of the miR-612 expression in tumor tissues. (H) Flow cytometry was used to detect the proportion of infiltrating DCs in tumor tissues. $* \mathrm{P}<0.05$, $* * \mathrm{P}<0.01$ and $* * * \mathrm{P}<0.00$ I.

miR-625 and thereby upregulating HMGA1. ${ }^{11}$ MetaLnc9 facilitates lung cancer metastasis via a PGK1-AKT/mTOR signaling pathway. ${ }^{24}$ LINC00963 is a predictor of poor prognosis and promotes esophageal cancer cell invasion via targeting the miR-214-5p/RAB14 axis. $^{25}$ LINC00963 facilitates osteosarcoma proliferation and invasion by suppressing the miR-204-3p/FN1 axis. ${ }^{26}$ Consistent with previous reports, the present results showed that LINC00963 overexpression in $\mathrm{GC}$ was related to the poor prognosis of patients with GC. In addition, knockdown of LINC00963 suppressed GC progression. Moreover, the maturation and function of DCs were repressed by the overexpression of LINC00963. These results suggested that LINC00963 was related to the progression of $\mathrm{GC}$ and may regulate $\mathrm{DC}$ mediated anti-tumor immune response.

LncRNAs serve as ceRNAs to regulate the expression of miRNAs and the progression of cancers. For instance, lncRNA UCA1 promotes malignant phenotypes of renal cancer cells by modulating the miR-182-5p/DLL4 axis. ${ }^{27}$ The lncRNA JPX/miR-33a-5p/Twist1 axis regulates the tumorigenesis and metastasis of lung cancer by activating Wnt/ $\beta$-catenin signaling. ${ }^{28}$ Long noncoding RNA LCAT1 functions as a ceRNA to regulate RAC1 function by sponging miR-4715-5p in lung cancer. ${ }^{29}$ However, the roles of LINC00963 in GC have not been elaborated. In the present study, miR-612 was a direct target of LINC00963, and this finding was confirmed with dualluciferase reporter assay. The expression of miR-612 was significantly downregulated in GC tissues and cell lines and negatively regulated by LINC00963. Functionally, overexpression of miR-612 inhibited the proliferation, EMT, migration, and invasion of GC cells. However, these results were completely reversed by LINC00963 overexpression. Consistent with this study, miR-612 also acts as a tumor suppressor in other cancers. For instance, miR-612 suppresses the stem cell-like property of 
A

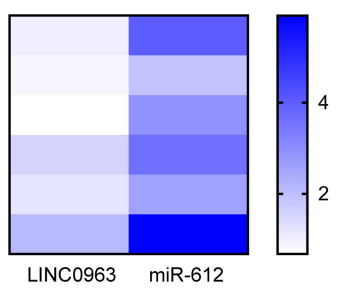

B

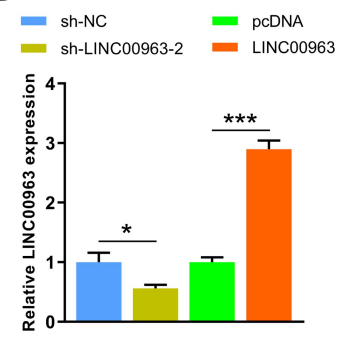

C

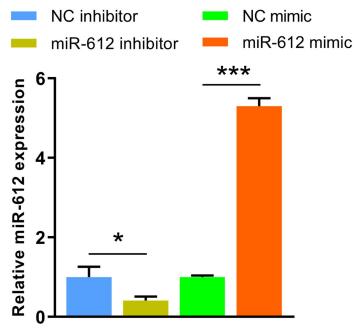

D

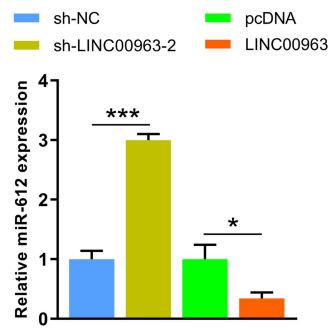

E

F

G
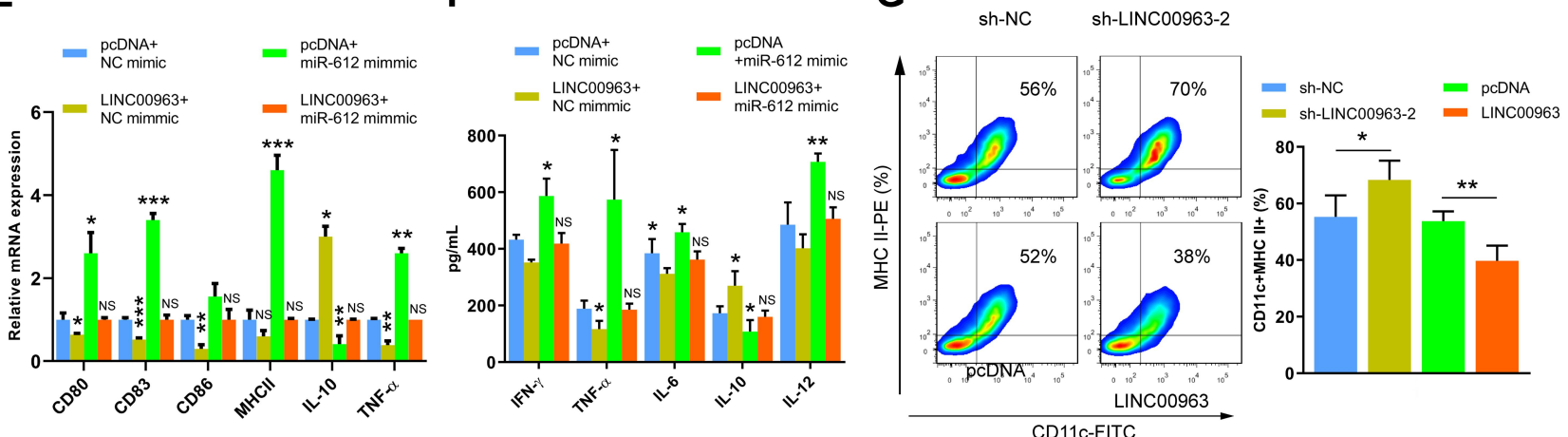

H
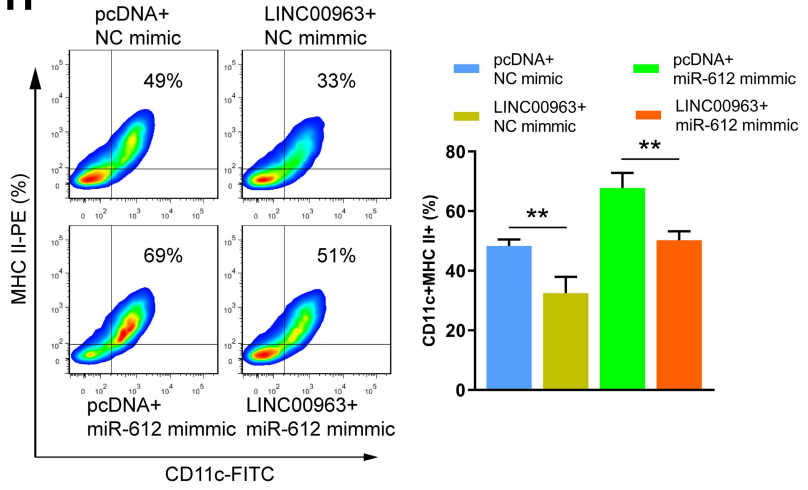

I

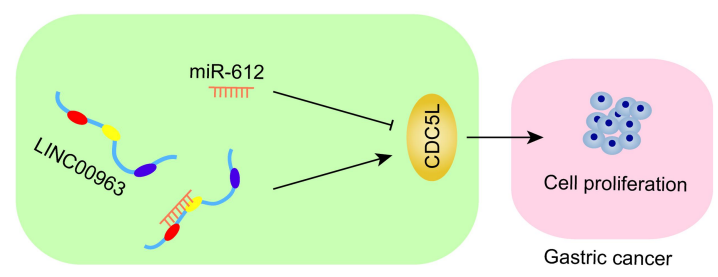

Figure 8 LINC00963 inhibited the differentiation and maturation of DCs in vitro. (A) Heat map analysis of miR-6I2 and LINC00963 expression in DCs. (B and C) Knockdown and overexpression of LINC00963 and miR-6I2 in DCs, respectively. (D) MiR-6I2 expression in DCs with low or high LINC00963 expression. (E) The expression of costimulatory markers (CD80, CD83, CD86, and MHCII) and cytokines (IL-10, TNF- $\alpha$ ) secreted from DC cells was analyzed by qRT-PCR. (F) The expression of cytokines (IFN- $\gamma$, TNF- $\alpha$, IL-6, IL-I0 and IL-I2) was analyzed by ELISA. (G) Flow cytometry was used to study the effects of LINC00963 during DCs maturation. (H) The opposite role of LINC00963 and miR-6I 2 was confirmed during DCs maturation by flow cytometry. (I) The flowchart of the study. $* \mathrm{P}<0.05$, $* * \mathrm{P}<0.0 \mathrm{I}$ and $* * * \mathrm{P}<0.00 \mathrm{I}$. Abbreviation: NS, not significant.

hepatocellular carcinoma cells by modulating Sp1/Nanog signaling. ${ }^{30}$ MiR-612 is downregulated and negatively regulates colorectal cancer growth and metastasis by targeting AKT2. ${ }^{31}$ MiR-612 suppresses the invasive-metastatic cascade in hepatocellular carcinoma. ${ }^{32}$ In addition, long noncoding RNA TRPM2-AS acts as a microRNA sponge of miR-612 to promote gastric cancer progression and radioresistance. ${ }^{33}$

CDC5L is a key regulator of cell division cycle and highly expressed in tumors. It is related to the progression of cancers. Previous studies found that CDC5L promotes
hTERT expression and colorectal tumor growth. ${ }^{34}$ CircPGAM1 promotes the malignant progression of epithelial ovarian cancer by regulating the miR-542-3p/CDC5L/ PEAK1 pathway. ${ }^{35}$ In addition, bladder cancer progression is inhibited because of CDC5L depletion. ${ }^{36}$ LncRNA NEAT1 accelerates prostate cancer progression by targeting CDC5L and regulating CDC5L-AGRN transcriptional regulation circuit. ${ }^{37} \mathrm{CDC} 5 \mathrm{~L}$ can also be used as a potential predictive biomarker for neo-adjuvant chemotherapy response in pediatric osteosarcoma. ${ }^{38}$ In the present study, bioinformatics software analysis and 
dual-luciferase reporter gene assay jointly confirmed that CDC5L is a target gene of miR-612. The occurrence and development of cancer are accompanied by changes in gene expression or alterations. We first analyzed and found the alterations of CDC5L in $\mathrm{GC}$, which hinted the importance of CDC5L in GC. Thus, CDC5L may be an indicator of GC diagnosis. We also found that the expression of CDC5L was abnormally elevated in GC cells. Furthermore, the roles of low CDC5L expression in the apoptosis, migration, and invasion of GC cells were abolished by LINC00963 overexpression. However, patients with altered levels of CDC5L exhibited slightly decreased overall survival from cBioPortal. This finding may be due to individual differences in samples or insufficient sample size.

LncRNAs play a key role during the differentiation and function of DCs. For instance, NEAT1 knockdown induces tolerogenic phenotype in dendritic cells by inhibiting activation of the NLRP3 inflammasome. ${ }^{16,39}$ In the present study, we reported for the first time that the proportion of DCs increased in the tumor tissue with low LINC00963 expression. Moreover, LINC00963 expression decreased in mature DCs. However, the expression of miR-612 was very high. Meanwhile, the expression of cytokines secreted from DCs was affected by LINC00963 and miR-612. In specific, LINC00963 inhibited the promoting effect of miR-612 on the differentiation and maturity of DCs in vitro.

\section{Conclusion}

In summary, our study indicated that LINC00963 was over-expressed in GC. Over-expression of LINC00963 accelerated proliferation, migration and invasion of $\mathrm{GC}$ cells, and inhibited DCs-related antitumor immune response. Furthermore, the promotion of LINC00963 on GC progression was achieved by competitively targeting miR-612 to upregulate CDC5L expression. Therefore, our study may expect to provide new insights into the treatment and diagnosis of GC.

\section{Acknowledgments}

This study was supported by Jiangsu Province Health and Planning Commission Scientific Research Guiding Project (NO. Z2017012).

\section{Author Contributions}

All authors made substantial contributions to conception and design, acquisition of data, or analysis and interpretation of data; took part in drafting the article or revising it critically for important intellectual content; agreed to submit to the current journal; gave final approval of the version to be published; and agree to be accountable for all aspects of the work.

\section{Disclosure}

The authors report no conflicts of interest in this work.

\section{References}

1. Siegel RL, Miller KD, Jemal A. Cancer statistics. CA Cancer J Clin. 2016;66(1):7-30.

2. Merchant SJ, Kim J, Choi AH, Sun V, Chao J, Nelson R. A rising trend in the incidence of advanced gastric cancer in young Hispanic men. Gastric Cancer. 2017;20(2):226-234. doi:10.1007/s10120-0160603-7

3. Ferro A, Peleteiro B, Malvezzi M, et al. Worldwide trends in gastric cancer mortality (1980-2011), with predictions to 2015, and incidence by subtype. Eur $J$ Cancer. 2014;50(7):1330-1344. doi:10.1016/j.ejca.2014.01.029

4. Tan P, Yeoh KG. Genetics and molecular pathogenesis of gastric adenocarcinoma. Gastroenterology. 2015;149(5):1153-62 e3. doi:10.1053/j.gastro.2015.05.059

5. Cancer Genome Atlas Research N. Comprehensive molecular characterization of gastric adenocarcinoma. Nature. 2014;513 (7517):202-209. doi:10.1038/nature 13480

6. Ashraf N, Hoffe S, Kim R. Adjuvant treatment for gastric cancer: chemotherapy versus radiation. oncologist. 2013;18(9):1013-1021. doi:10.1634/theoncologist.2012-0462

7. Zhang C, Ma MH, Liang Y, Wu KZ, Dai DQ. Novel long non-coding RNA LINC02532 promotes gastric cancer cell proliferation, migration, and invasion in vitro. World J Gastrointest Oncol. 2019;11 (2):91-101. doi:10.4251/wjgo.v11.i2.91

8. Abraham JM, Meltzer SJ. Long noncoding RNAs in the pathogenesis of barrett's esophagus and esophageal carcinoma. Gastroenterology. 2017;153(1):27-34. doi:10.1053/j.gastro.2017.04.046

9. Liu K, Wang BJ, Han W, et al. CFIm25-regulated lncRNA acv3UTR promotes gastric tumorigenesis via miR-590-5p/YAP1 axis. Oncogene. 2020;39(15):3075-3088. doi:10.1038/s41388-020-1213-8

10. Wang CJ, Zhu CC, Xu J, et al. The IncRNA UCA1 promotes proliferation, migration, immune escape and inhibits apoptosis in gastric cancer by sponging anti-tumor miRNAs. Mol Cancer. 2019;18(1):115. doi:10.1186/s12943-019-1032-0

11. Wu Z, Wang W, Wang Y, et al. Long noncoding RNA LINC00963 promotes breast cancer progression by functioning as a molecular sponge for microRNA-625 and thereby upregulating HMGA1. Cell Cycle. 2020;19(5):610-624. doi:10.1080/15384101.2020.1728024

12. Liu W, Yang YJ, An Q. LINC00963 promotes ovarian cancer proliferation, migration and EMT via the miR-378g/CHI3L1 Axis. Cancer Manag Res. 2020;12:463-473. doi:10.2147/CMAR.S229083

13. Jiao H, Jiang S, Wang H, Li Y, Zhang W. Upregulation of LINC00963 facilitates melanoma progression through miR-608/ NACC1 pathway and predicts poor prognosis. Biochem Biophys Res Commun. 2018;504(1):34-39. doi:10.1016/j.bbrc.2018.08.115

14. Wu JH, Tian XY, An QM, Guan XY, Hao CY. LINC00963 promotes hepatocellular carcinoma progression by activating PI3K/AKT pathway. Eur Rev Med Pharmacol Sci. 2018;22(6):1645-1652.

15. Wculek SK, Cueto FJ, Mujal AM, Melero I, Krummel MF, Sancho D. Dendritic cells in cancer immunology and immunotherapy. Nat Rev Immunol. 2020;20(1):7-24. doi:10.1038/ s41577-019-0210-z 
16. Wang P, Xue Y, Han Y, et al. The STAT3-binding long noncoding RNA lnc-DC controls human dendritic cell differentiation. Science. 2014;344(6181):310-313. doi:10.1126/science.1251456

17. Xiang Z, Zhou ZJ, Xia GK, et al. A positive crosstalk between CXCR4 and CXCR2 promotes gastric cancer metastasis. Oncogene. 2017;36(36):5122-5133. doi:10.1038/onc.2017.108

18. Chi Y, Wang D, Wang J, Yu W, Yang J. Long non-coding RNA in the pathogenesis of cancers. Cells. 2019;8:9. doi:10.3390/cells8091015

19. Shuai Y, Ma Z, Liu W, et al. TEAD4 modulated LncRNA MNX1-AS1 contributes to gastric cancer progression partly through suppressing BTG2 and activating BCL2. Mol Cancer. 2020;19(1):6.

20. Yari H, Jin L, Teng L, et al. LncRNA REG1CP promotes tumorigenesis through an enhancer complex to recruit FANCJ helicase for REG3A transcription. Nat Commun. 2019;10(1):5334. doi:10.1038/ s41467-019-13313-z

21. Liu PY, Tee AE, Milazzo G, et al. The long noncoding RNA lncNB1 promotes tumorigenesis by interacting with ribosomal protein RPL35. Nat Commun. 2019;10(1):5026. doi:10.1038/s41467-01912971-3

22. Taiana E, Favasuli V, Ronchetti D, et al. Long non-coding RNA NEAT1 targeting impairs the DNA repair machinery and triggers anti-tumor activity in multiple myeloma. Leukemia. 2020;34 (1):234-244. doi:10.1038/s41375-019-0542-5

23. Qin G, Tu X, Li H, et al. Long noncoding RNA p53-stabilizing and activating RNA promotes p53 signaling by inhibiting heterogeneous nuclear ribonucleoprotein $\mathrm{k}$ desumoylation and suppresses hepatocellular carcinoma. Hepatology. 2020;71(1):112-129. doi:10.1002/ hep. 30793

24. Yu T, Zhao Y, Hu Z, et al. MetaLnc9 facilitates lung cancer metastasis via a pgk1-activated akt/mTOR pathway. Cancer Res. 2017;77 (21):5782-5794. doi:10.1158/0008-5472.CAN-17-0671

25. Liu HF, Zhen Q, Fan YK. LINC00963 predicts poor prognosis and promotes esophageal cancer cells invasion via targeting miR-214-5p/ RAB14 axis. Eur Rev Med Pharmacol Sci. 2020;24(1):164-173.

26. Zhou Y, Yin L, Li H, Liu LH, Xiao T. The LncRNA LINC00963 facilitates osteosarcoma proliferation and invasion by suppressing miR-204-3p/FN1 axis. Cancer Biol Ther. 2019;20(8):1141-1148. doi:10.1080/15384047.2019.1598766

27. Wang W, Hu W, Wang Y, et al. Long non-coding RNA UCA1 promotes malignant phenotypes of renal cancer cells by modulating the miR-182-5p/DLL4 axis as a ceRNA. Mol Cancer. 2020;19(1):18. doi:10.1186/s12943-020-1132-x

28. Pan J, Fang S, Tian H, et al. IncRNA JPX/miR-33a-5p/Twist1 axis regulates tumorigenesis and metastasis of lung cancer by activating Wnt/beta-catenin signaling. Mol Cancer. 2020;19(1):9. doi:10.1186/ s12943-020-1133-9
29. Yang J, Qiu Q, Qian X, et al. Long noncoding RNA LCAT1 functions as a ceRNA to regulate RAC1 function by sponging miR-4715-5p in lung cancer. Mol Cancer. 2019;18(1):171. doi:10.1186/s12943-0191107-y

30. Liu Y, Liu DL, Dong LL, et al. miR-612 suppresses stem cell-like property of hepatocellular carcinoma cells by modulating Sp1/Nanog signaling. Cell Death Dis. 2016;7(9):e2377. doi:10.1038/ cddis.2016.282

31. Sheng L, He P, Yang X, Zhou M, Feng Q. miR-612 negatively regulates colorectal cancer growth and metastasis by targeting AKT2. Cell Death Dis. 2015;6:e1808. doi:10.1038/cddis.2015.184

32. Tao ZH, Wan JL, Zeng LY, et al. miR-612 suppresses the invasive-metastatic cascade in hepatocellular carcinoma. $J$ Exp Med. 2013;210(4):789-803. doi:10.1084/jem.20120153

33. Xiao J, Lin L, Luo D, et al. Long noncoding RNA TRPM2-AS acts as a microRNA sponge of miR-612 to promote gastric cancer progression and radioresistance. Oncogenesis. 2020;9(3):29.

34. Li J, Zhang N, Zhang R, et al. CDC5L promotes htert expression and colorectal tumor growth. Cellular Physiol Biochem. 2017;41 (6):2475-2488. doi:10.1159/000475916

35. Zhang C, Li Y, Zhao W, Liu G, Yang Q. Circ-PGAM1 promotes malignant progression of epithelial ovarian cancer through regulation of the miR-542-3p/CDC5L/PEAK1 pathway. Cancer Med. 2020;9 (10):3500-3521. doi:10.1002/cam4.2929

36. Zhang Z, Mao W, Wang L, et al. Depletion of CDC5L inhibits bladder cancer tumorigenesis. $J$ Cancer. 2020;11(2):353-363. doi: $10.7150 /$ jca. 32850

37. Li X, Wang X, Song W, et al. Oncogenic properties of neat1 in prostate cancer cells depend on the cdc5l-agrn transcriptional regulation circuit. Cancer Res. 2018;78(15):4138-4149. doi:10.1158/00085472.CAN-18-0688

38. Martin JW, Chilton-MacNeill S, Koti M, van Wijnen AJ, Squire JA, Zielenska M. Digital expression profiling identifies RUNX2, CDC5L, MDM2, RECQL4, and CDK4 as potential predictive biomarkers for neo-adjuvant chemotherapy response in paediatric osteosarcoma. PLoS One. 2014;9(5):e95843. doi:10.1371/journal.pone.0095843

39. Zhang M, Zheng Y, Sun Y, et al. Knockdown of NEAT1 induces tolerogenic phenotype in dendritic cells by inhibiting activation of NLRP3 inflammasome. Theranostics. 2019;9(12):3425-3442. doi: $10.7150 /$ thno. 33178
OncoTargets and Therapy

\section{Publish your work in this journal}

OncoTargets and Therapy is an international, peer-reviewed, open access journal focusing on the pathological basis of all cancers, potential targets for therapy and treatment protocols employed to improve the management of cancer patients. The journal also focuses on the impact of management programs and new therapeutic

Submit your manuscript here: https://www.dovepress.com/oncotargets-and-therapy-journal agents and protocols on patient perspectives such as quality of life, adherence and satisfaction. The manuscript management system is completely online and includes a very quick and fair peer-review system, which is all easy to use. Visit http://www.dovepress.com/ testimonials.php to read real quotes from published authors. 